\title{
Simultaneous Determination of Elastic Constants and Asphericity of Spherical Specimens by the Resonant Sphere Technique
}

\author{
Hitoshi Oda \\ Department of Earth Sciences, Okayama University, Okayama 700, Japan
}

\begin{abstract}
A method is developed to simultaneously determine both the elastic constants and asphericity of a spherical specimen by the resonant sphere technique (RST). First, a numerical test is presented to examine the possibility of simultaneous determination of the specimen's elastic constants and asphericity by an inversion analysis of the resonant frequencies measured for the specimen. Then, the method is applied to elasticity measurements of a spherical olivine specimen with small asphericity. The simultaneous inversion analysis method allows one to evaluate elastic constants and asphericity more accurately than the previous method, in which the asphericity and elastic properties are separately determined. Since the asphericity of a spherical specimen has a noticeable influence on the calculated elastic constants, simultaneous inversion is an effective method to obtain improvements in the accuracy of the elastic constants and to quantify the asphericity of the specimen by the RST.
\end{abstract}

\section{Introduction}

The measurement of elastic constants is important for studying the Earth's interior because the composition and structure of the Earth can be investigated by comparing laboratory data of elastic properties with elasticity profiles obtained from seismic studies. Thus, a number of ultrasonic methods have been developed to precisely measure the elastic constants of candidate materials (e.g., McSkimin, 1964; Fraser and Lecraw, 1964; Anderson et al., 1969; Demarest, 1971; Ohno, 1976).

The resonant sphere technique (RST), first developed by Fraser and Lecraw (1964), is one method that has been extensively used in making elasticity measurements (e.g., Soga and Anderson, 1967; Suzuki et al., 1992a; Oda et al., 1992, 1994a). In the RST, the resonant frequencies of a spherical specimen are measured. The elastic constants are then determined by minimizing, in a least squares sense, the differences between the measured frequencies and frequencies calculated from a set of elastic constants by assuming a perfect sphere. However, even when care is extended in attempting to shape the specimen into a perfect sphere, there is always some asphericity associated with the prepared specimen. The problem is magnified when making measurements of an anisotropic material at high temperatures since anisotropic thermal expansion causes

Received July 8, 1996; Accepted April 17, 1997 
further deviations from the perfect sphericity of the specimen's shape. In such cases, the effect of asphericity on the measured resonant frequency spectrum should be corrected because, in the RST, the elastic constants are determined under the assumption of a perfectly spherical specimen. In view of these complications, Oda et al. (1994b) and Oda (1996) examined the relative shift in frequency due to asphericity by comparing the resonant frequencies of ellipsoidal and spherical specimens for the same oscillation mode. They showed that the relative frequency shifts are of the same order in magnitude as the asphericity of the ellipsoid. These results suggest that the asphericity of an ellipsoidal specimen, as well as the elastic constants, may be determined by the RST if shifts in resonant frequencies due to asphericity are sufficiently larger than the uncertainties in the measured frequencies.

In this study, we develop a method to simultaneously determine both the elastic constants and asphericity of an elastically anisotropic ellipsoid. An application is then made to an olivine specimen that has orthorhombic crystal symmetry.

\section{Method}

Consider an ellipsoid of a single-crystal material that has orthogonal crystal axes. When the major axes of the ellipsoid are parallel to the crystal axes, the difference $(\delta f)$ in a particular resonant frequency between the ellipsoid and a reference sphere with a volume equal to that of the ellipsoid is approximated by $\delta f=\Psi_{x} \varepsilon_{x}+\Psi_{y} \varepsilon_{y}+\Psi_{z} \varepsilon_{z}$ (Oda, 1996), where $\varepsilon_{i}$ and $\Psi_{i}(i=x, y, z)$ are the asphericity and aspherical coefficients of the ellipsoid, respectively. The aspherical coefficients $\left(\Psi_{i}\right)$ represent how sensitive a resonant frequency $(f)$ is to a small change in asphericity (i.e., $\left.\Psi_{i}=\partial f / \partial \varepsilon_{i}\right)$. The asphericity $\left(\varepsilon_{i}\right)$ is defined by

$$
\varepsilon_{x}=\left(d_{x}-\hat{a}\right) / \hat{a}, \quad \varepsilon_{y}=\left(d_{y}-\hat{a}\right) / \hat{a}, \quad \varepsilon_{z}=\left(d_{z}-\hat{a}\right) / \hat{a},
$$

where $d_{x}, d_{y}$, and $d_{z}$ are the lengths of the semi-major axes of the ellipsoid in the $x, y$, and $z$ directions. In Eq. (1), $\hat{a}$ is the radius of the reference sphere and equals the average of $d_{x}, d_{y}$, and $d_{z}$. Since the volume of the ellipsoid is equal to that of the reference sphere, a constraint on the asphericity is obtained: $\varepsilon_{x}+\varepsilon_{y}+\varepsilon_{z}=0$.

In addition to the frequency shift due to asphericity, recall that a resonant frequency is also affected by the elastic properties of the specimen. In order to simultaneously determine the elastic constants and asphericity of the ellipsoidal specimen by an iterative inversion procedure, we approximated the difference $(\delta f)$ of an observed frequency from its calculated value. Since the calculated value is based on initial assumptions regarding the elastic properties and the asphericities of the specimen, in general,

$$
\delta f=\Psi_{i} \delta \varepsilon_{i}+B_{i j} \delta C_{i j}, \quad(i, j=x, y, z)
$$

where the summation convention is assumed for repeated indices. The terms labelled $\delta \varepsilon_{i}$ and $\delta C_{i j}$ on the righthand side of Eq. (2) are the contributions from small corrections to the assumed values of asphericity and elasticity, respectively, of the specimen. The coefficients ( $\Psi_{i}$ and $B_{i j}$ ) are easily evaluated using analytic expressions (Oda, 1996; Oda et al., 1993), provided that the frequencies of the resonance modes are computed for given values of $\varepsilon_{i}$ and $C_{i j}$. We accomplished this using the XYZ algorithm developed by 
Visscher et al. (1991). If $\delta \varepsilon_{i}$ and $\delta C_{i j}$ are simultaneously determined by solving Eq. (2) for a set of observed resonance modes by a least squares calculation, then a refined set of elastic constants and asphericity values is obtained by adding these small corrections to the initial values of $\varepsilon_{i}$ and $C_{i j}$. The inversion is repeated by using the corrected values as the initial values of the elastic constants and asphericity until the root mean square (RMS) of the differences between observed and computed frequencies converges to a specified small value.

\section{Numerical Test}

Before applying the present method to elasticity measurements of an olivine specimen, we present the results of a numerical test that was performed using artificial data of eigenfrequencies generated from an ellipsoidal olivine. The elastic constants and asphericity are given in the first column of Table 1 . The goal of the test was to confirm whether or not the elastic constants and asphericity could be obtained by an inversion analysis of the artificial eigenfrequency data shown in the bottom spectrum in Fig. 1. The initial values of the elastic constants and asphericity for the inversion are listed in the second column of Table 1 . The specimen was initially assumed to be a sphere with a radius of $0.893 \mathrm{~mm}$. The frequencies computed for the initial values of the perfect sphere are compared with the artificial frequencies in the middle row of Fig. 1. The RMS of the differences between the artificial and computed frequencies was $17 \mathrm{kHz}$. The frequency residuals are attributed to the small differences between the artificial and initial values of the elastic constants and asphericity. Thus, information regarding the

Table 1. Physical parameters of the olivine sphere in the numerical test.

Crystal symmetry: Orthorhombic

Density $\left(\mathrm{g} / \mathrm{cm}^{3}\right): 3.35$

Radius, $\hat{a}(\mathrm{~cm}): 0.0893$

\begin{tabular}{lccr}
\hline & Artificial* & Initial** & Final*** \\
\hline Asphericity & & & \\
$\varepsilon_{x}$ & 0.0 & 0.0 & 0.0 \\
$\varepsilon_{y}$ & 0.01 & 0.0 & 0.01 \\
$\varepsilon_{z}$ & -0.01 & 0.0 & -0.01 \\
Elastic constants $C_{i j}(\mathrm{GPa})$ & & & \\
$C_{11}$ & 319.61 & 320.0 & 319.61 \\
$C_{22}$ & 197.32 & 200.0 & 197.32 \\
$C_{33}$ & 236.84 & 240.0 & 236.84 \\
$C_{44}$ & 64.06 & 65.0 & 64.06 \\
$C_{55}$ & 77.76 & 78.0 & 77.76 \\
$C_{66}$ & 78.34 & 79.0 & 78.34 \\
$C_{23}$ & 75.67 & 77.0 & 75.67 \\
$C_{31}$ & 70.92 & 72.0 & 70.92 \\
$C_{12}$ & 69.24 & 71.0 & 69.24 \\
\hline
\end{tabular}

\footnotetext{
* Values of $C_{i j}$ and $\varepsilon_{i}$ set to make the artificial data of resonant frequencies. ${ }^{* *}$ Initial values of $C_{i j}$ and $\varepsilon_{i}$ *** Final values of $C_{i j}$ and $\varepsilon_{i}$ determined during the fourth iteration.
}

Vol. 45, No. 4, 1997 

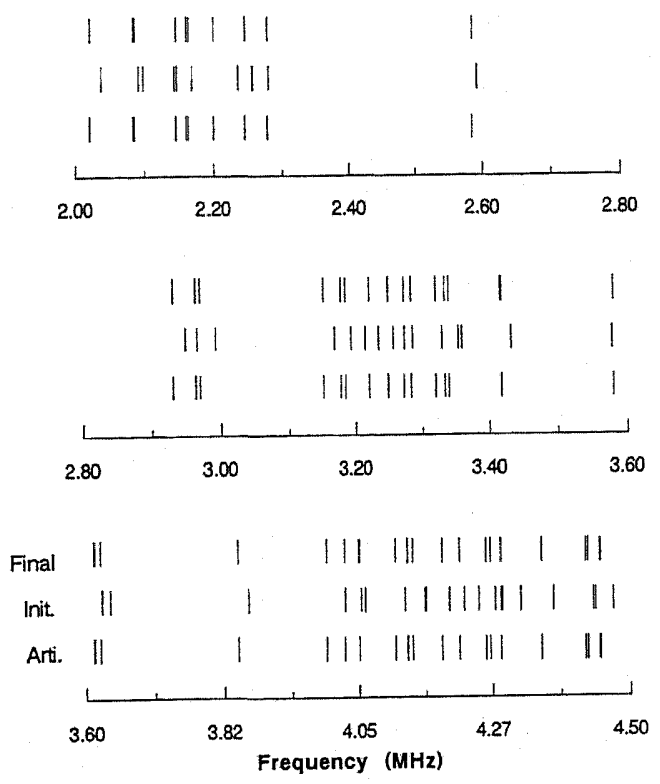

Fig. 1. Comparison between the artificial and computed frequencies in the numerical test. The bottom spectrum (Arti.) shows the artificial resonant frequencies. The middle (Init.) and top (Final) spectra represent the theoretical frequencies computed for the initial and final values, respectively, of elastic constants and asphericity. The lowest 45 resonance modes are shown in the spectra.

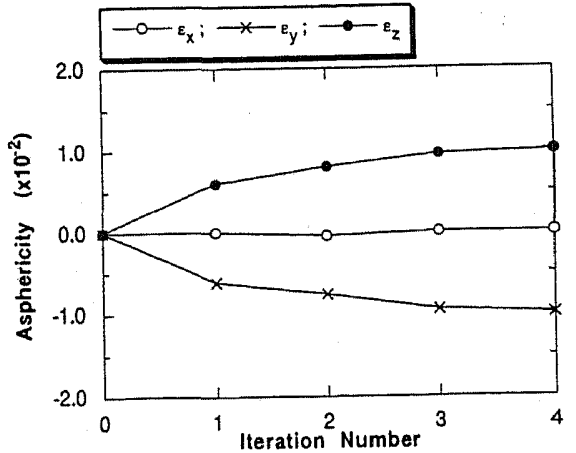

Fig. 2. Plot of asphericity versus iteration number in the numerical test. The final values of asphericity agree exactly with the artificial ones at the fourth iteration.

elastic constants and asphericity is contained in the frequency differences.

The inversion was repeated four times to obtain the final values for elastic constants and asphericity. There was a perfect agreement between the artificial and final sets of values (see Table 1). Figure 2 depicts the change of asphericity against the iteration number. The values of asphericity gradually approached the artificial values as the 
inversion was repeated. In Fig. 3, the differences between the initial and determined values of the elastic constants are plotted against the iteration number. The RMS of the frequency residuals was about $17 \mathrm{kHz}$ at the initial stage of inversion but decreased with increasing iteration number and vanished at the fourth iteration (see Fig. 4). These numerical results point to the possibility of simultaneously determining both the asphericity and elastic constants of an ellipsoidal specimen by inverting the resonant frequencies.

According to a numerical experiment by Isoda (1989), relative differences of the order of $10^{-4}$ in resonant frequencies give changes smaller than $0.1 \mathrm{GPa}$ in the elastic constants determined by a least squares calculation for spherical specimens. Similarly, a small change of $10^{-4}$ in asphericity $\left(\delta \varepsilon_{i}\right)$ produces relative frequency shifts of the same order in magnitude as the change in asphericity (Oda, 1996). Thus, resonant frequencies of the order of $10^{6} \mathrm{~Hz}$ should be measured to an accuracy within $100 \mathrm{~Hz}$ in order to determine the elastic constants and asphericity within probable errors smaller than $0.1 \mathrm{GPa}$ and $10^{-3}$, respectively.
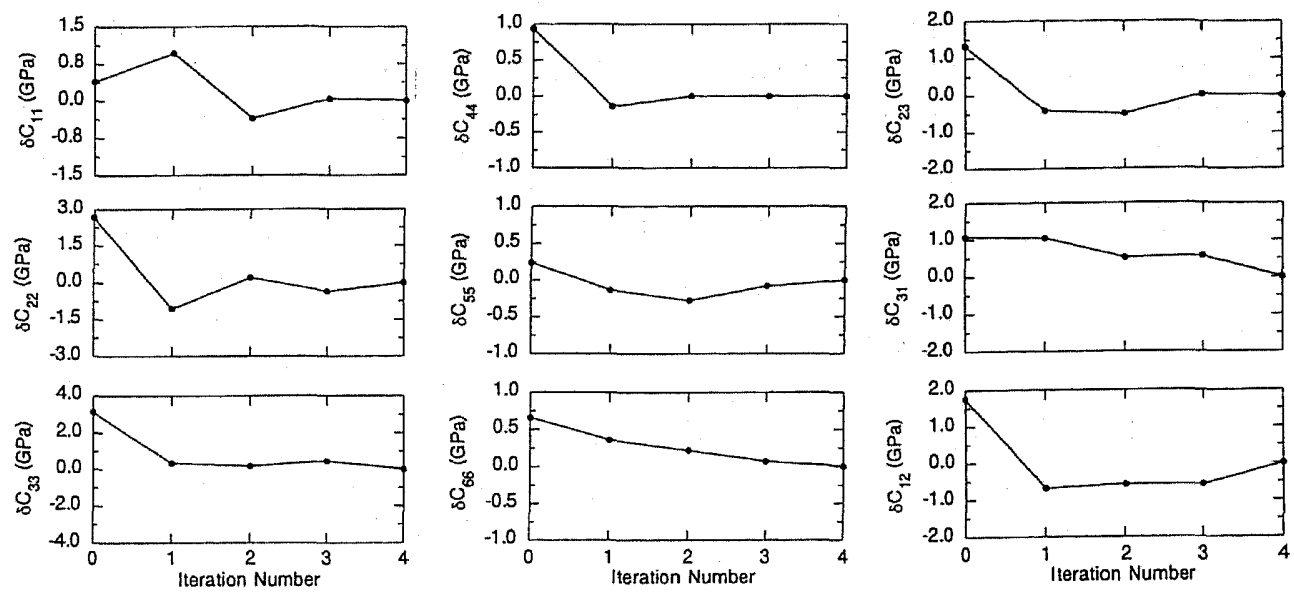

Fig. 3. Differences between the determined and artificial elastic constants versus iteration number. When the differences vanish, the determined elastic constants agree perfectly with the artificial ones.

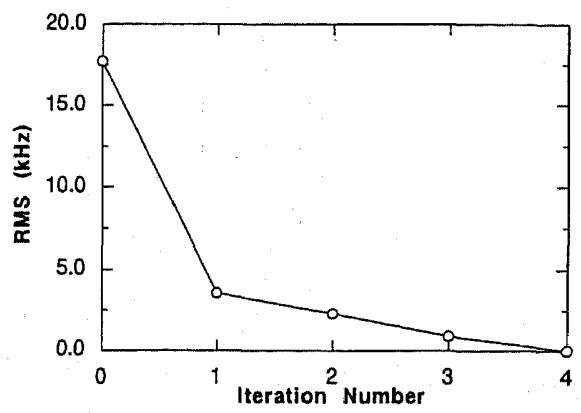

Fig. 4. Plot of the RMS of the frequency residuals versus iteration number.

Vol. 45, No. 4, 1997 


\section{Application}

The present method was applied to a spherical olivine specimen with small asphericity. The density and radius of the specimen are given in Table 2 . The resonant frequencies of the specimen were measured to an accuracy better than $100 \mathrm{~Hz}$ by Suzuki et al. (1992b). Here, we use these data in a simultaneous inversion for elastic constants and asphericity of the specimen. We adopted the elastic constants which Oda and Visscher (1994) reported from these same frequency data as the initial values of $C_{i, j}$ 's. This data reduction was made under the assumption of a perfect sphere. The initial values of the elastic constants are given in Table 2. In Fig. 5, the measured frequencies of the lowest 42 resonance modes are compared with frequencies calculated for the initial values of the elastic constants. Good agreement was found between the measured and computed frequencies, but small differences remained between both sets of frequencies. The RMS of the frequency residuals was $2.8 \mathrm{kHz}$.

Assuming that the differences between the measured and computed frequencies are due to the asphericity of the specimen, two kinds of inversions were made to determine the asphericity: an inversion for obtaining the asphericity alone, and an inversion for obtaining the asphericity and elastic constants simultaneously. In the first inversion, the second term in the righthand side of Eq. (2) was omitted, and thus the number of unknown parameters was three. In the second inversion scheme, there were twelve unknown parameters since the olivine had nine independent elastic constants. The initial values of the model parameters listed in Table 2 were used as the starting point for

Table 2. Physical parameters of the olivine sphere.

\begin{tabular}{lrrr}
\hline $\begin{array}{l}\text { Crystal symmetry: Orthorhombic } \\
\text { Density }\left(\mathrm{g} / \mathrm{cm}^{3}\right): 3.35 \\
\text { Radius, } \hat{a}(\mathrm{~cm}): 0.0893\end{array}$ & & & \\
& & & \\
\hline & Initial* & 1st inv.** & 2nd inv. ${ }^{* *}$ \\
\hline Asphericity $\left(\times 10^{-3}\right)$ & & & \\
$\varepsilon_{x}$ & 0.0 & $1.07 \pm 0.23$ & $2.11 \pm 0.12$ \\
$\varepsilon_{y}$ & 0.0 & $1.02 \pm 0.28$ & $0.88 \pm 0.13$ \\
$\varepsilon_{z}$ & 0.0 & $-2.09 \pm 0.36$ & $-2.99 \pm 0.18$ \\
Elastic constants $C_{i j}$ (GPa) & & & $322.88 \pm 0.30$ \\
$C_{11}$ & $319.61 \pm 0.61$ & - & $196.61 \pm 0.19$ \\
$C_{22}$ & $197.32 \pm 0.57$ & - & $235.07 \pm 0.21$ \\
$C_{33}$ & $236.84 \pm 0.60$ & - & $63.86 \pm 0.02$ \\
$C_{44}$ & $64.06 \pm 0.06$ & - & $77.88 \pm 0.03$ \\
$C_{55}$ & $77.76 \pm 0.08$ & - & $78.52 \pm 0.03$ \\
$C_{66}$ & $78.34 \pm 0.08$ & - & $74.69 \pm 0.11$ \\
$C_{23}$ & $75.67 \pm 0.34$ & - & $71.52 \pm 0.12$ \\
$C_{31}$ & $70.92 \pm 0.36$ & - & $69.29 \pm 0.12$ \\
$C_{12}$ & $69.24 \pm 0.38$ & - & \\
\hline
\end{tabular}

* The initial values of elastic constants are as determined by Oda and Visscher (1994) on the assumption of a spherical olivine with a radius of $0.0893 \mathrm{~cm} .{ }^{* *}$ The final values obtained in the first and second inversions are shown in the columns of 1 st inv. and 2 nd inv., respectively. 


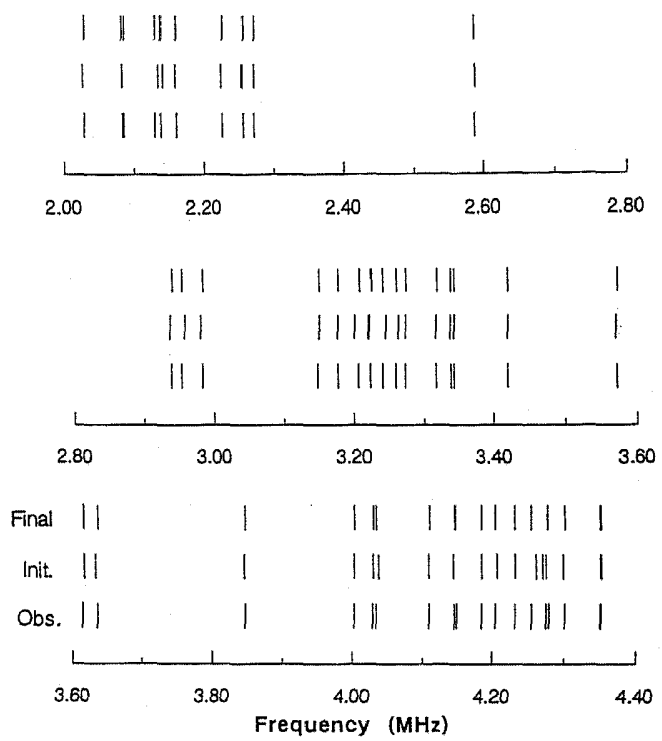

Fig. 5. Comparison between the measured and computed frequencies of an olivine sphere using simultaneous inversion. The lowest 42 resonance modes are identified by the RST. The bottom spectrum (Obs.) shows the measured resonant frequencies. The middle (Init.) and top (Final) spectra represent the theoretical frequencies computed for the initial and final values of elastic constants and asphericity, respectively.

both inversions. Three iterations were sufficient in each case to achieve convergence of the frequencies as seen by the small RMS of the frequency residuals.

The final values of asphericity and elastic constants of the olivine specimen are listed in Table 2 together with their probable errors, which were obtained in solving Eq. (2) by a least squares calculation. The probable errors of the determined asphericity are smaller in the simultaneous inversion than in the inversion only for asphericity. Figure 6 shows the changes of asphericity against the iteration number in the first and second inversions. The asphericity of the specimen was almost completely determined during the first iteration of the inversion and was of the order of $10^{-3}$ in magnitude. The specimen's shape was ellipsoidal with long major axes in the $x$ and $y$ directions and a short axis in the $z$ direction. This result is also supported by other experimental evidence. Suzuki et al. (1992b) suggested, by direct measurement of the radius of the same spherical specimen, that the specimen's shape may be approximated by a spheroid $\left(d_{x}=d_{y} \neq d_{z}\right)$ with a short axis in the $z$ direction.

The elastic constants obtained in the second inversion are shown as a function of the iteration number in Fig. 7. The theoretical frequencies computed for the final values of the elastic constants are shown in the top spectrum in Fig. 5. The RMS of the frequency differences was $2.8 \mathrm{kHz}$ before executing the inversion but decreased to $1.8 \mathrm{kHz}$ when inverting the frequency data only for asphericity, and further decreased to below $1.0 \mathrm{kHz}$ by simultaneous inversion for elastic constants and asphericity (see Fig. 8).

Vol. 45, No. 4, 1997 

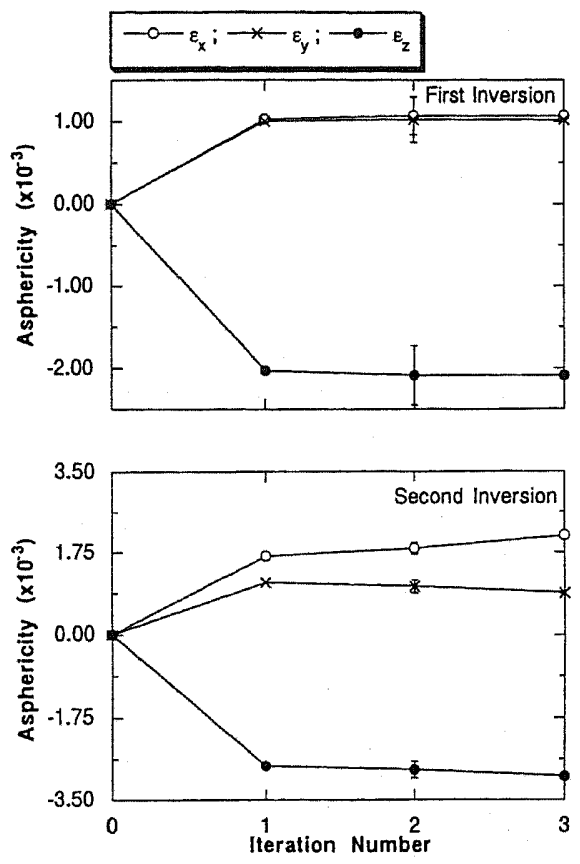

Fig. 6. Plot of asphericity versus iteration number for the first and second inversions. The vertical bars represent the probable error in asphericity determined during the second iteration.
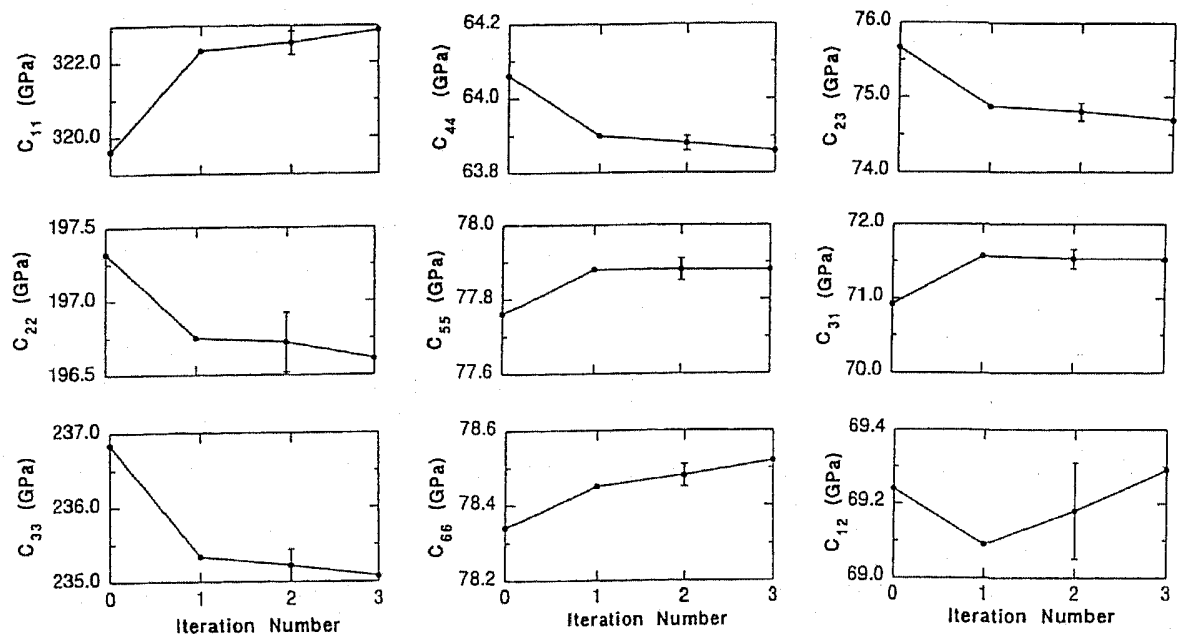

Fig. 7. Plot of elastic constants versus iteration number using simultaneous inversion for elastic constants and asphericity. The vertical bars represent the probable error in elastic constants determined at the second iteration. 


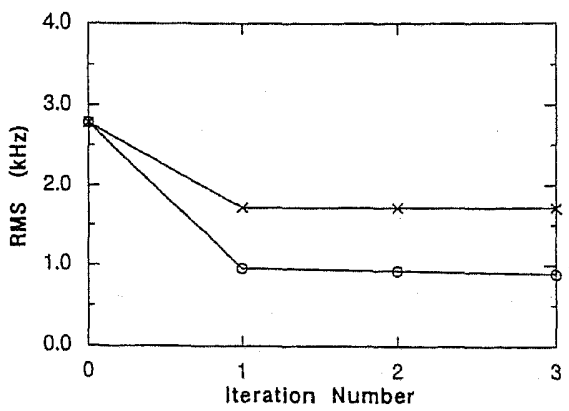

Fig. 8. Plot of the RMS of the frequency residuals versus iteration number in the inversion of asphericity only (cross) and simultaneous inversion of elastic constants and asphericity (circle).

Such an excellent agreement between the measured and computed frequencies shows that simultaneous inversion provides elastic constants which are more accurate than those obtained by assuming a perfectly spherical specimen. We note that the probable errors of our redetermined elastic constants are smaller than those of the initial elastic constants determined by the inversion of elastic constants only (see Table 2). The absolute differences between the initial and final values of elastic constants were smaller than $1 \%$, but generally outside the overlap of errors. However, a small RMS of $0.9 \mathrm{kHz}$ still remained between the measured and computed frequencies. This small disagreement may be attributable to a tilt between the major axes and the crystal axes of the olivine, a slight deviation of the specimen's shape from truly ellipsoidal, measurement errors of the resonant frequencies, imperfections or inhomogeneities in the olivine crystal, and so on.

\section{Discussion}

We have compared the inversions of frequency data that assume perfectly spherical specimens and allow for deviations from perfect spheres in order to show how the calculated elastic constants would change as a result of the simultaneous inversion of both elasticity and specimen geometry. It was found that changes in elastic constants smaller than $1 \%$ were produced by asphericity of the order of $10^{-3}$. Thus, asphericity has a small but noticeable effect on the determination of elastic constants by the RST. We then considered how a tilt between the major and crystal axes of an ellipsoidal olivine specimen with small asphericity would affect the accuracy of elastic constants determined by a least squares method. Oda (1994) investigated the effect of tilt upon the resonant frequencies of an olivine sphere with an asphericity of the order of $10^{-3}$, and showed that the tilt gave frequency shifts of a few kilohertzes for resonant frequencies that are in the $10^{6} \mathrm{~Hz}$ range. Because these frequency shifts are nearly equal to shifts resulting from an asphericity of $10^{-3}$, the tilt between the ellipsoidal and crystal axes may cause further changes that are slightly smaller than the $1 \%$ change in elastic constants due to asphericity. 


\section{Conclusion}

In order to correct the influence of asphericity on elasticity measurements of a spherical specimen by the RST, a method of simultaneous determination of the elastic constants and asphericity was presented. A numerical test was performed to demonstrate that it is possible to simultaneously determine both elastic constants and asphericity by the inversion of resonant frequencies. The method was applied to a spherical olivine specimen with small asphericity. We found that the specimen had an asphericity of the order of $10^{-3}$. The elastic constants determined from the simultaneous inversion of both the $C_{i j}$ 's and asphericity differed from the elastic constants determined under the assumption of a perfectly spherical geometry by about $1 \%$ or less. The elastic constants and asphericity were more accurately determined by simultaneous inversion than by the individual inversion of elastic constants or asphericity. Simultaneous inversion is an effective method for obtaining improved accuracy of elastic constants and for quantifying the asphericity of spherical solids by means of the RST.

The author thanks Naoto Yoshioka, Don Isaak, and an anonymous reviewer for giving helpful comments to revise the manuscript.

\section{REFERENCES}

Anderson, D. L., G. C. Sammis, and R. A. Phinney, Brillouin scattering-a new geophysical tool, in The Application of Modern Physics to the Earth and Planetary Interiors, ed. S. K. Runcorn, pp. 465-477, Wiley-Interscience, London, 1969.

Demarest, H. H., Cube-resonance method to determine the elastic constants of solids, J. Acoust. Soc. Am., 49, 768-775, 1971.

Fraser, D. B. and R. C. Lecraw, Novel method of measuring elastic and anelastic properties of solids, Rev. Sci. Instr., 35, 1113-1115, 1964.

Isoda, S., Computation of free oscillation eigenfrequency of elastically anisotropic spheres: a base for the resonant sphere technique, Master thesis of Okayama University, pp. 1-144, 1989.

McSkimin, H. J., Ultrasonic method for measuring the mechanical properties of a liquid and solids, in Physical Acoustics, Vol. 1A, ed. W. P. Mason, pp. 272-335, Academic Press, New York, 1964.

Oda, H., Free oscillation of an elastically anisotropic ellipsoid with arbitrary crystal orientation, J. Phys. Earth, 42, 97-101, 1994.

Oda, H., Aspherical correction for free vibration of elastically anisotropic solid by means of the XYZ algorithm, Pure Appl. Geophys., 147, 719-727, 1996.

Oda, H. and W. M. Visscher, Comparison between SEF and XYZ algorithms for computation of eigenfrequencies of elastically anisotropic solid, J. Seismol. Soc. Jpn., 47, 97-100, 1994 (in Japanese).

Oda, H., I. Suzuki, and I. Ohno, Partial derivatives of eigenfrequencies of a rectangular parallelepiped and a sphere of elastically anisotropic solid, J. Phys. Earth, 41, 271-289, 1993.

Oda, H., O. L. Anderson, D. G. Isaak, and I. Suzuki, Measurement of elastic properties of single-crystal $\mathrm{CaO}$ up to $1200 \mathrm{~K}$, Phys. Chem. Min., 19, 96-105, 1992. 
Oda, H., S. Isoda, Y. Inouye, and I. Suzuki, Elastic constants and anelastic properties of an anisotropic periclase sphere as determined by the resonant sphere technique, J. Geophys. Res., 99, 15517-15527, 1994a.

Oda, H., J. Hirao, I. Suzuki, W. M. Visscher, and O. L. Anderson, Free oscillations of elastically anisotropic spheres and ellipsoids, Geophys. J. Int., 118, 555-565, 1994b.

Ohno, I., Free vibration of a rectangular parallelepiped crystal and its application to determination of elastic constants of orthorhombic crystals, J. Phys. Earth, 24, 355-379, 1976.

Soga, N. and O. L. Anderson, Elastic properties of tektites measured by resonant sphere technique, J. Geophys. Res., 72, 1733-1739, 1967.

Suzuki, I., H. Oda, S. Isoda, T. Saito, and K. Seya, Free oscillation of an elastically anisotropic sphere and its application to determining the elastic constants of rutile, J. Phys. Earth, 40, 601-616, 1992a.

Suzuki, I., Y. Inouye, J. Hirao, H. Oda, T. Saito, and K. Seya, The resonant sphere technique for measurements of elasticity and anelasticity of a small mineral specimen-an application to olivine, J. Seismol. Soc. Jpn., 45, 213-228, 1992 b (in Japanese).

Visscher, W. M., A. Migliori, T. M. Bell, and R. A. Reinert, On the normal modes of free vibration of inhomogeneous and anisotropic elastic objects, J. Acoust. Soc. Am., 90, 2154-2162, 1991.

Vol. 45 , No. 4, 1997 